\title{
Flavonolignans inhibit the arachidonic acid pathway in blood platelets
}

Michal Bijak ${ }^{*}$ and Joanna Saluk-Bijak

\begin{abstract}
Background: Arachidonic acid metabolism by cyclooxygenase (COX) is a major pathway for blood platelets' activation, which is associated with pro-thrombotic platelet activity and the production of pro-inflammatory mediators. Inhibition of COX activity is one of the major means of anti-platelet pharmacotherapy preventing arterial thrombosis and reducing the incidence of cardiovascular events. Recent studies have presented that a silymarin (standardized extract of Milk thistle (Silybum marianum)) can inhibit the COX pathway. Accordingly, the aim of our study was to determine the effects of three major flavonolignans (silybin, silychristin and silydianin) on COX pathway activity in blood platelets.

Methods: We determined the effect of flavonolignans on arachidonic acid induced blood platelet aggregation, COX pathway metabolites formation, as well as COX activity in platelets. Additionally, we analysed the potential mechanism of this interaction using the bioinformatic ligand docking method.
\end{abstract}

Results: We observed that tested compounds decrease the platelet aggregation level, both thromboxane $\mathrm{A}_{2}$ and malondialdehyde formation, as well as inhibit the COX activity. The strongest effect was observed for silychristin and silybin. In our in silico study we showed that silychristin and silybin have conformations which interact with the active COX site as competitive inhibitors, blocking the possibility of substrate binding.

Conclusions: The results obtained from this study clearly present the potential of flavonolignans as novel antiplatelet and anti-inflammatory agents.

Keywords: Flavonolignans, Silybin, Silychristin, Silymarin, Arachidonic acid, Blood platelet, Cyclooxygenase

\section{Background}

Blood platelets are the smallest, un-nucleated morphotic elements of human blood that play a major role in the blood coagulation system. The biological activity of platelets, both in physiological processes as well as under pathological conditions, is dependent on the degree of their activation. A platelet's activation process, despite the absence of a nucleus, is very complex and associated with elements of enzymatic signal transduction chains [1]. After the platelets' activation, signal transduction leads to mobilization of intracellular calcium ions $\left(\mathrm{Ca}^{2+}\right)$. High intracellular concentration of $\mathrm{Ca}^{2+}$ results in activation of phospholipases, which are responsible for the release of cell membrane phospholipids' enzymatic hydrolyses. These include, for example, the precursor of

\footnotetext{
* Correspondence: michal.bijak@biol.uni.lodz.pl

Department of General Biochemistry, Faculty of Biology and Environmental

Protection, University of Lodz, Pomorska 141/143, 90-236 Lodz, Poland
}

essential bioactive eicosanoids - 5,8,11,14-eicosatetraenoic acid called arachidonic acid (AA), which is a 20carbon polyunsaturated fatty acid. AA released from the membranes is enzymatically oxidized, transformed by the cyclic peroxide prostaglandin synthase known as cyclooxygenase (COX) into intermediate products: proinflammatory prostaglandins and pro-thrombotic thromboxane $\mathrm{A}_{2}\left(\mathrm{TXA}_{2}\right)[2,3]$. These are accompanied by production of reactive oxygen species (ROS) [4]. TXA 2 is generated from prostaglandin $\mathrm{H} 2$, formed by $\mathrm{COX}$ through thromboxane-A synthase. TXA 2 is an autocrine or paracrine mediator in the nearby tissues surrounding its production site. $\mathrm{TXA}_{2}$ is a very strong blood platelet activator acting as a pro-aggregator and vasoconstrictor mediator, leading to increased platelet aggregation. This plays a pivotal role in the growth and stabilization of a coronary thrombus [5]. $\mathrm{TXA}_{2}$ is formed in platelets in 
response to local stimuli and exerts an activating effect within a short distance of its biosynthesis.

AA metabolism by COX is a major pathway of blood platelets activation, and is associated with pro-thrombotic platelets' activity and the production of pro-inflammatory mediators. AA addition in vitro to platelet rich plasma causes a burst of oxygen consumption, $\mathrm{TXA}_{2}$ generation and platelet aggregation [6].

One of the major method in anti-platelet pharmacotherapy of preventing arterial thrombosis is inhibition of COX activity. The results of clinical studies have shown that intake of aspirin, or different aspirin-like COXinhibitors, reduces the incidence of cardiovascular events [7]. Low-dose aspirin (40 mg per day) supplementation reduces the risk of serious cardiovascular events by $12 \%$ and non-fatal myocardial infarction by $18 \%$. This dose is able to inhibit a large proportion of thromboxane $\mathrm{A}_{2}$ release provoked acutely by the platelets' response. Aspirin is also able to reduce the risk of secondary thrombotic events by about $25 \%$ [8].

Experiments performed on human monocytes have shown that flavonolignans - active chemical compounds presented in a silymarin (standardized extract from of Milk thistle (Silybum marianum)) inhibit the COX pathway [9]. In accordance with this observation, the aim of our study was to determine the effects of three major flavonolignans (silybin, silychristin and silydianin) on COX pathway activity in blood platelets.

\section{Methods}

\section{Reagents}

Dimethyl sulfoxide (DMSO), 3-[(3-Cholamidopropyl)dimethylammonio]-1-propanesulfonate (CHAPS), 4-(2Hydroxyethyl)piperazine-1-ethanesulfonic acid (HEPES), glucose, trichloroacetic acid, thiobarbituric acid, Tris, flavonolignans (silybin, silychristin and silydianin) were all obtained from the Sigma-Aldrich Chemical Co. (St. Louis, MO, USA). Arachidonic acid was purchased from Chrono-Log (Havertown, PA USA). All other chemicals were reagent grade or the highest-quality available.

\section{Blood samples}

Blood samples collected from different healthy donors were purchased from the Regional Center for Transfusion Medicine in Lodz (Poland). All samples were drawn in the morning, from fasting donors. All donors were checked by a medical doctor and found to have no cardiovascular disorders, allergy, lipid or carbohydrate metabolism disorders, nor were they being treated with any drugs [10]. Our analysis of the blood samples was performed under the guidelines of the Helsinki Declaration for Human Research, and approved by the Committee on the Ethics of Research in Human Experimentation at the University of Lodz (Resolution No. 16/KBBN-UŁ/II/2016).

\section{Isolation of platelet-rich-plasma and blood platelets}

The blood was centrifuged $(200 \times g, 10 \mathrm{~min}, \mathrm{RT})$ to isolate the platelet rich plasma (PRP). The obtained PRP was then used to measure aggregation and platelet isolation. Blood platelets were isolated by differential centrifugation of blood, as described above [11, 12]. The final concentration of platelet suspension was about $4 \times 10^{8}$ platelets $/ \mathrm{ml}$. The platelets were counted using a photometric method according to Walkowiak et al. [13]. The platelet washing procedure was performed in plastic tubes and carried out at room temperature. Washed human platelets were suspended in a modified Tyrode's $\mathrm{Ca}^{2+} / \mathrm{Mg}^{2+}$ free buffer $(127 \mathrm{mM} \mathrm{NaCl}, 2.7 \mathrm{mM} \mathrm{KCl}$, $0.5 \mathrm{mM} \mathrm{NaH} \mathrm{PO}_{4}, 12 \mathrm{mM} \mathrm{NaHCO}, 5 \mathrm{mM}$ HEPES, $5.6 \mathrm{mM}$ glucose, $\mathrm{pH} 7.4$ ).

The isolated platelets, as well as the PRP samples, were pre-incubated with flavonolignans (silybin, silychristin and silydianin) in a concentration range of $10-100 \mu \mathrm{M}$ by $30 \mathrm{~min}$ at $37^{\circ} \mathrm{C}$. All tested compounds were initially dissolved in $20 \%$ DMSO to a preliminary concentration of $20 \mathrm{mM}$. Other solutions of the compounds used were also performed in 20\% DMSO (prepared in $50 \mathrm{mM}$ TBS, $\mathrm{pH}$ 7.4). The final DMSO concentration of all samples was $0.1 \%$. In control samples the same volume of solvent was added (20\% DMSO prepared in $50 \mathrm{mM}$ TBS, pH 7.4), with the probes warmed for $30 \mathrm{~min}$ at $37^{\circ} \mathrm{C}$. The isolated and purified blood platelets were used to determine the level of TBARS and activity of COX. Samples dedicated to the determination of COX activity were dissolved in 1:1 Cell Lysis Buffer (BD Pharmingen ${ }^{\mathrm{Tm}}$ ).

\section{Platelet aggregation induced by AA}

Platelet aggregation was measured turbidimetrically in PRP using the optical Chrono-Log aggregometer (Chrono-Log, Havertown, PA). The PRP samples were pre-incubated with flavonolignans (silybin, silychristin and silydianin) at the concentration range of $10-100 \mu \mathrm{M}$ by $30 \mathrm{~min}$ at $37^{\circ} \mathrm{C}$. All tested compounds were initially dissolved in $20 \%$ DMSO (prepared in $50 \mathrm{mM}$ TBS, $\mathrm{pH}$ 7.4) to the preliminary concentration of $20 \mathrm{mM}$. The final concentration of DMSO in all samples was $0.1 \%$. In the control samples, the same volume of solvent was added (20\% DMSO prepared in $50 \mathrm{mM}$ TBS, $\mathrm{pH}$ 7.4), with the probes warmed for $30 \mathrm{~min}$ at $37^{\circ} \mathrm{C}$. The prepared PRP samples were prewarmed at $37{ }^{\circ} \mathrm{C}$ and stirred. After the pre-incubation procedure for the PRP samples, the free AA (1 mM) were added and platelet aggregation measured for $10 \mathrm{~min}$. The aggregometer was calibrated each time (100\% aggregation) on platelet poor plasma (PPP), with the appropriate concentration of each flavonolignan. 


\section{Determination of COX-1 activity}

The level of COX-1 activity in the platelet lysates was determined by the fluorescence-based method, using a COX Fluorescent Activity Assay Kit (Cayman Chemicals). The oxygenase activity of COX causes conversion of the arachidonic acid to a prostaglandin $\mathrm{G} 2\left(\mathrm{PGG}_{2}\right)$ which is the first intermediate in the COX pathway. This assay is based on the reaction formed between $\mathrm{PGG}_{2}$ and 10-acetyl-3,7dihydroxyphenoxazine (ADHP), which produces the highly fluorescent compound Resorufin. This can be analysed using an excitation wavelength of $530-540 \mathrm{~nm}$ and an emission wavelength of 585-595 $\mathrm{nm}$. The activity of COX is presented herein as the amount of Resorufin produced per time unit $[\mathrm{nmol} / \mathrm{min}]$. Each step of procedure was performed according to manufacturer protocol.

\section{Estimation of thiobarbituric acid reactive substances}

Samples of blood platelet suspended in the modified Tyrode's buffer were treated with thrombin $(1 \mathrm{U} / \mathrm{ml})$. After $10 \mathrm{~min}$ the samples were mixed with an equal volume of $15 \%(w / v)$ cold trichloroacetic acid, and with an equal volume of $0.37 \%(w / v)$ thiobarbituric acid in $0.25 \mathrm{M} \mathrm{HCl}$. All samples were immersed in a boiling water bath for $10 \mathrm{~min}$. After cooling, the samples were centrifuged and then absorbance was measured at $535 \mathrm{~nm}$. The results were estimated using a molar extinction coefficient of malondialdehyde (MDA), a reliable marker of lipid peroxidation $\left(\varepsilon=1.56 \times 10^{5} \mathrm{M}\right.$ $\left.{ }^{-1} \mathrm{~cm}^{-1}\right)$, and expressed as a percent of control value, as described previously [14].

\section{Determination of the level of $\mathrm{TXB}_{2}$ using a competitive ELISA assay}

All of the blood samples were pre-incubated with flavonolignans (silybin, silychristin and silydianin), in a concentration range of $10-100 \mu \mathrm{M}$ for $30 \mathrm{~min}$ at $37^{\circ} \mathrm{C}$. In the control samples, the same volume of stock flavonolignans solvent was added (20\% DMSO prepared in $50 \mathrm{mM}$ TBS, $\mathrm{pH}$ 7.4), with the probes warmed for $30 \mathrm{~min}$ at $37^{\circ} \mathrm{C}$. After that, the samples were transferred into Sarstedt S-Monovette Serum tubes with a coagulation activator (thrombin $2.5 \mathrm{U} / \mathrm{ml}$ ), and left at $37{ }^{\circ} \mathrm{C}$. After $30 \mathrm{~min}$ the samples were centrifuged $(4500 \mathrm{rpm}$, $15 \mathrm{~min}, 37^{\circ} \mathrm{C}$ ) to obtain serum. To quantify the level of Thromboxane $\mathrm{B} 2\left(\mathrm{TXB}_{2}\right)$ in the samples (the serum was diluted 100 times), a Thromboxane $B_{2}$ Express ELISA Kit - Monoclonal (Cayman Chemicals) was used. The total level of $\mathrm{TXB}_{2}$ in all samples was obtained as $\mathrm{ng} / \mathrm{ml}$ using a calibration curve and expressed as a percent of control value.

\section{Ligand docking}

The ligand dockings for the selected flavonolignans to the cyclooxygenase were calculated in silico with Autodock
Vina 1.0, an algorithm released by Scripps Research Institute [15] (http://vina.scripps.edu/), in accordance with the previously used procedure [16-18]. The protein coordinates and PDB format structure of the cyclooxygenase-1 $3 \mathrm{~N} 8 \mathrm{Z}$ [19] were taken from the website of the RSCB Protein Data Bank (http://www.rcsb.org). All threedimensional chemical structures of the flavonolignans were downloaded from PubChem (http://pubchem.ncbi.nlm.nih.gov/) and converted to.pdb files using Avogadro 1.1.0, an open-source molecular builder and visualization tool (http://avogadro.openmolecules.net/) [20]. Protein preparation for the docking procedure was performed in a Swiss-PdbViewer (http://spdbv.vital-it.ch/). The nonbonded atoms present in the crystal structure were removed. Next, the receptor structure was adapted in Auto Dock Tools v 1.5.6rc1 (http://autodock.scripps.edu) [21] and the missing hydrogen atoms, Gasteiger charges [22] as well as Kollman [23] united atom charges were calculated and assigned. Non-polar hydrogens were merged, and rotatable bonds assigned, keeping all the amide bonds as non-rotatable. The protein file was prepared in.pdbqt format, which is pdb plus "q" charges and the " $\mathrm{t}$ " AutoDock type. The docking procedure was run with the following settings: center_x - 21.039; center_y 53.126; center_z 10.252; size_x 126; size_y 126; size_z 126 . The binding points were computed and the binding affinity of the ligand to the receptor counted in $\mathrm{kcal} / \mathrm{mol}$. Analysis and visualization of the three-dimensional structures of the protein with the bound ligand was performed with the Python Molecular Viewer of Auto Dock Tools v 1.5.6rc1 (http://autodock.scripps.edu) [21], and Swiss-PdbViewer (http://spdbv.vital-it.ch/) [24]. The docking protocol procedure was validated using flurbiprofen compound, which was docked to the same place as presented in the crystal structure. This indicated the reliability of the presented docking method.

\section{Data analysis}

The statistical analysis was performed using StatsDirect statistical software V. 2.7.2. All experimental values presented in this study were expressed as mean \pm standard deviation (SD). To analyse the normality of the distribution of results, the Shapiro-Wilk test was used. Next, the results were analysed under the equality of variance using Levene's test. The significance of the differences between the values was analysed using ANOVA, followed by Tukey's range test for multiple comparisons (for data with normal distribution and equality of variance), and the Kruskal-Wallis test; $p<0.05$ was accepted as statistically significant [25-28].

\section{Results}

In the first step of our study we examined the effect of flavonolignans on COX pathway-induced blood platelets 
aggregation. To ensure full platelet activation, a physiological stimulus was added - AA (1 mM). In our measurements we found that in a dose-dependent manner, all tested flavonolignans inhibit the activation of platelets in PRP upon AA stimulation, resulting in a statistically significant dose-dependent decrease in the platelet aggregation level. The strongest inhibitory effects were observed for silychristin and silybin, which at the highest used concentration $(100 \mu \mathrm{M})$ reduced AA induced blood platelets aggregate formation (control value of $75 \%$ ), to $10 \%$ and $18 \%$ respectively. In the samples treated with silydianin we also observed an inhibitory effect (Fig. 1). However, it was lower in comparison to the silybin and silychristin.

Next, we established the effect of flavonolignans on the generation of the main COX pathway metabolite: thromboxane A2. Generation of $\mathrm{TXA}_{2}$ was determined by measurement of the concentration of its stable metabolite $\mathrm{TXB}_{2}$, using the ELISA method. In all samples where blood had been treated with flavonolignans, a reduction of $\mathrm{TXB}_{2}$ concentration was observed (Fig. 2). In samples treated with silychristin and silybin $(100 \mu \mathrm{M})$, the levels of generated thromboxane were about $75 \%$ and $60 \%$ reduced, respectively. In the case of the highest concentration of silydianin, the level of generated thromboxane was reduced by about 35\%. Our results also revealed that in a dose-dependent manner, flavonolignans inhibit synthesis of AA metabolites, measured as the amount of TBARS and expressed as nmoles of MDA per $\mathrm{ml}$ of platelet suspension (Fig. 3).

In the next step of our study, we determined the direct effect of flavonolignans on the oxygenase activity of COX1 , measured in blood platelets. We observed that all examined flavonolignans caused the inhibition of COX activity (Fig. 4). COX inhibition by the tested compounds was expressed as a $\mathrm{IC}_{50}$ value - the concentration needed to inhibit $50 \%$ of enzyme activity. The strongest inhibitory effect was demonstrated by silychristin and silybin $\left(\mathrm{IC}_{50}\right.$ for silychristin was $35 \mu \mathrm{M}, 60 \mu \mathrm{M}$ for silybin), while for silydianin we did not obtain an $\mathrm{IC}_{50}$ value (at the maximum tested concentration of $100 \mu \mathrm{M}$, a 32\% inhibition of COX activity was observed).

In our in silico study we computer-generated models of interaction between COX and selected flavonolignans (silychristin and silybin). We observed that both tested compounds have conformities which interact with active site of COX, blocking the possibility of substrates binding. B rings of Silychristin and silybin (Fig. 5) bind to the entry of active site loop where is located Tyr385 residue. The obtained affinity parameters demonstrate that silychristin and silybin have a strong binding mode to COX active site entry $(-9.8 \mathrm{kcal} / \mathrm{mol}$ and $-9.2 \mathrm{kcal} / \mathrm{mol}$ respectively), in comparison to flurbiprofenum, one of the most popular non-steroid anti-inflammatory drugs $(-8.9 \mathrm{kcal} / \mathrm{mol})$.

\section{Discussion}

Cyclooxygenase is a representative of membrane-bound glycoproteins and possesses two distinct enzymatic activities: oxygenase and peroxidase. In the early 1990s, identification of two members of the COX family was made. COX-1 is constitutively expressed in many types of cells, however it is the only COX form expressed in blood platelets. COX-2 is an inducible form which is activated in cells by pro-inflammatory inflammatory cytokines [29].

COX-pathway activation in blood platelets is strictly dependent on its substrate - the unesterified free form of arachidonic acid released from the internal phospholipid membrane [30]. AA is the main fatty acid component of phospholipids in blood platelets' cell layer (phosphatidylcholine, phosphatidylethanolamine and phosphatidylinositol

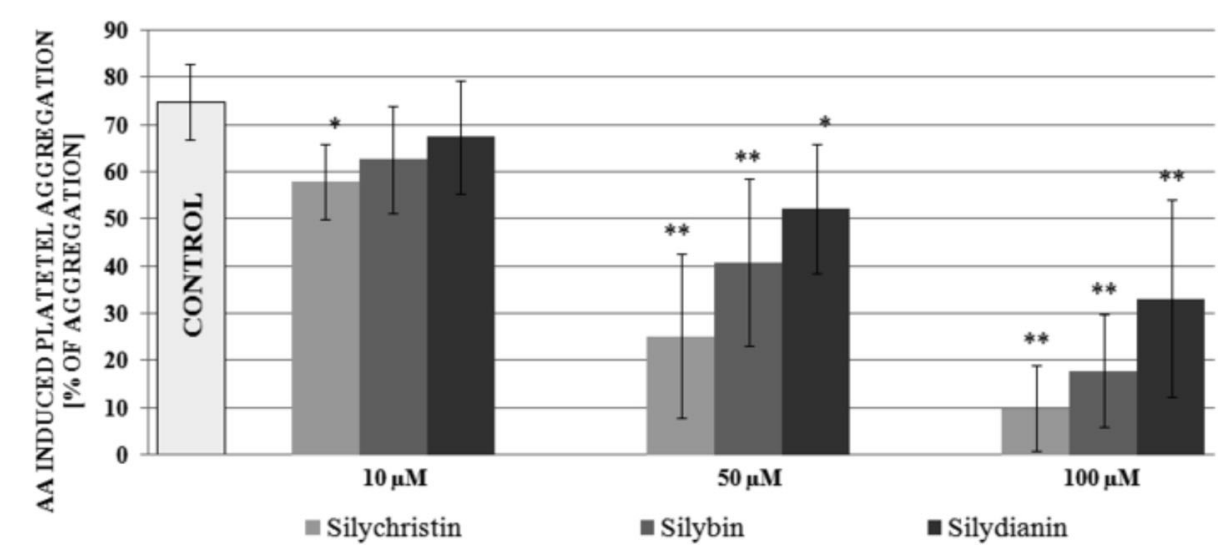

Fig. 1 The effect of flavonolignans (silychristin, silybin and silydianin in concentrations of 10; 50 and $100 \mu \mathrm{M}$ ) on blood platelet aggregation induced by arachidonic acid in platelet-rich plasma. The data represents means of $\pm S D, n=12$. Statistical analysis was performed using Tukey's Range Test, ${ }^{*} p<0.05,{ }^{* *} p<0.001$ 


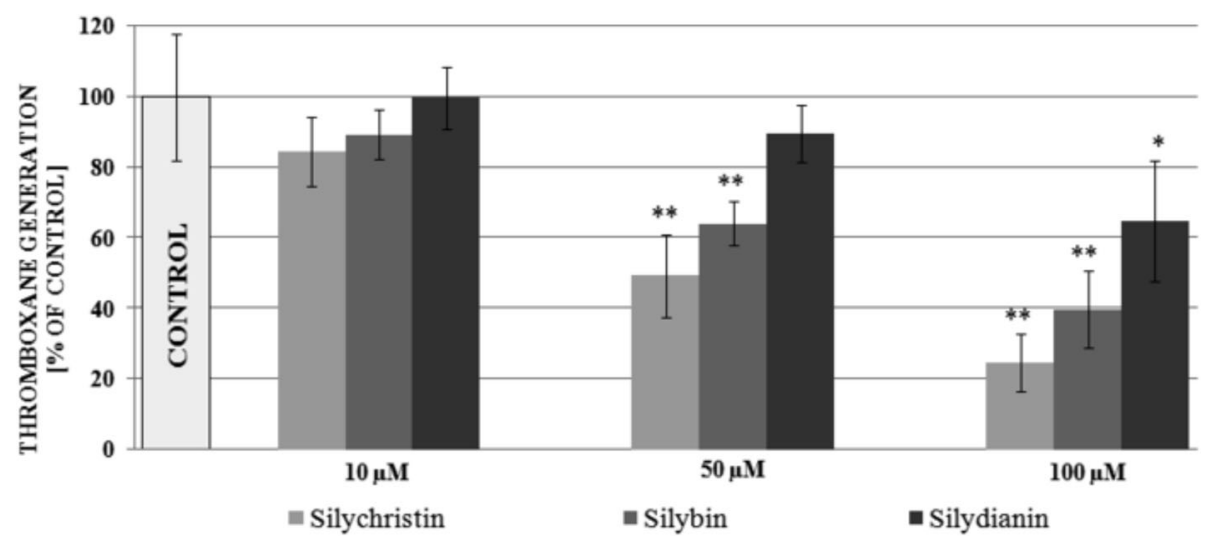

Fig. 2 The effect of flavonolignans (silychristin, silybin and silydianin at concentrations 10; 50 and $100 \mu \mathrm{M}$ ) on TXA 2 generation in blood platelets. The concentration of $\mathrm{TXA}_{2}$ metabolite $\left(\mathrm{TX}_{2}\right)$ was measured in serum obtained after blood coagulation activation by thrombin. The data represents means of $\pm S D, n=12$. Statistical analysis was performed using Tukey's Range Test ${ }^{*} p<0.05,{ }^{* *} p<0.001$

may contain even $80 \%$ of AA) [2]. After being released by phospholipase $\mathrm{A}_{2}$ and phospholipase $\mathrm{C}$, free $\mathrm{AA}$ is oxidized at $\mathrm{C}-11$ by COX. The oxygenase activity of COX catalyses the incorporation of two oxygen atoms to one molecule of arachidonate, creating a prostaglandin $\mathrm{G} 2\left(\mathrm{PGG}_{2}\right)$. Next, peroxidase catalysis results in reduction of the $15-\mathrm{OOH}$ group in the $\mathrm{PGG}_{2}$ structure to the $15-\mathrm{OH}$ group, and formation of prostaglandin $\mathrm{H} 2\left(\mathrm{PGH}_{2}\right) . \mathrm{PGH}_{2}$ is a substrate for enzymes in the heme prosthetic group - thromboxane A (TXA) synthase. The heme group presented in the catalytic centre of TXA synthase is responsible for homolytic cleavage of the epidioxy bond in $\mathrm{PGH}_{2}$, and rearrangement to TXA $_{2}$ [31].

One of the direct actions of thromboxane on blood platelets is to modify the response of platelets to exogenous agonists, as well as to stimulate platelet activation. The platelet's response to $\mathrm{TXA}_{2}$ is responsible for the surface receptor for $\mathrm{TXA}_{2}$ (TP), which is a member of the seven-transmembrane G-protein-coupled receptor family. Activation of TP results in both the autocrine and paracrine action of $\mathrm{TXA}_{2}$ released from blood platelets. The TP signalling pathway is mediated by two $G$ proteins: $G_{q}$ and $G_{12 / 13} . G_{q}$ is responsible for activation of phospholipase $C$, resulting in increased inositol 1,4,5-triphosphate concentration. In consequence, intra-cellular $\mathrm{Ca}^{2+}$ ions are mobilised and diacylglycerol is formed, which activates protein kinase $C$. These events cause platelet shape change, aggregation, and secretion from intra-cellular granules. Stimulation of $\mathrm{G}_{12 / 13}$ subunits activates the Rho pathway, which results in myosin light chain phosphorylation and subsequent platelet shape change [32].

$\mathrm{TXA}_{2}$ is a very unstable molecule with a short half-life of about $30 \mathrm{~s}$, after which it passes to the very stable but inactive metabolite $\mathrm{TXB}_{2}$ [32].

The involvement of blood platelets in both the physiological coagulation process and pathological conditions

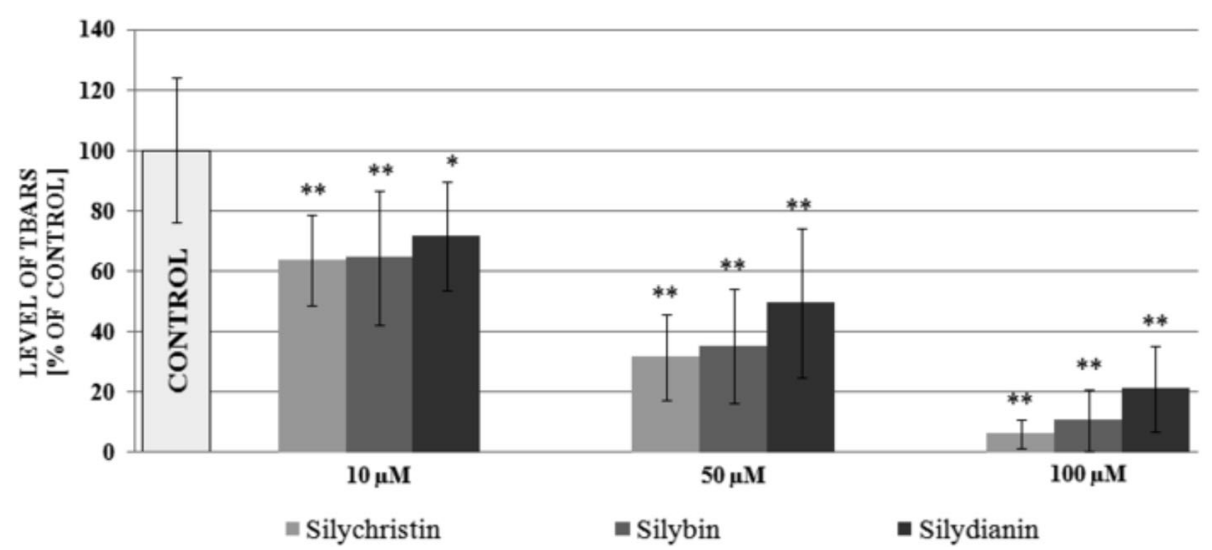

Fig. 3 The effect of flavonolignans (silychristin, silybin and silydianin at concentrations 10; 50 and $100 \mu \mathrm{M}$ ) on the blood platelets COX metabolite pathway was estimated by the level of TBARS concentration measurement. The data represents means of $\pm S D, n=12$. Statistical analysis was performed using the Kruskal-Wallis test, ${ }^{*} p<0.05,{ }^{* *} p<0.001$ 


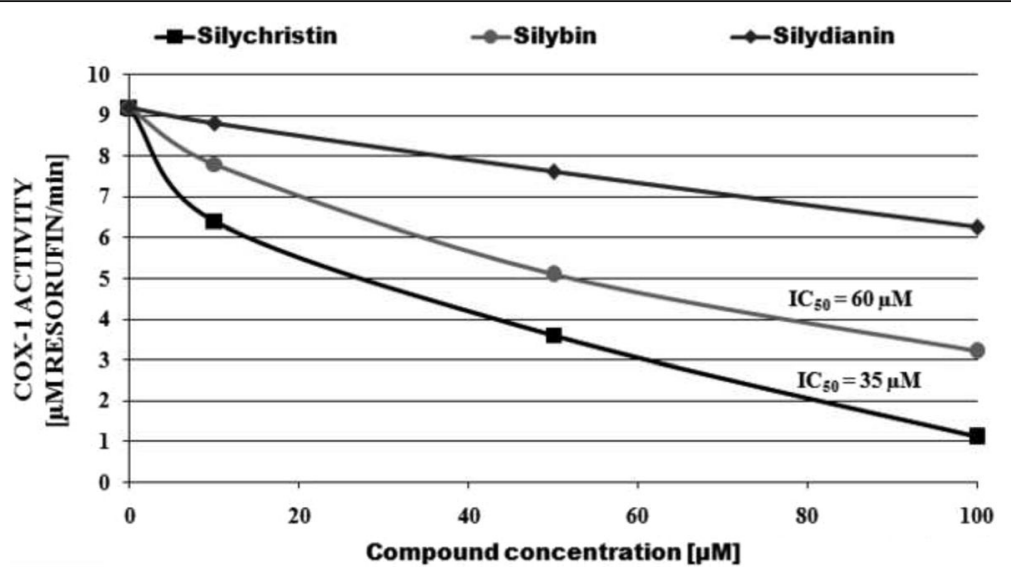

Fig. 4 The effect of flavonolignans (silychristin, silybin and silydianin at concentrations 10; 50 and $100 \mu \mathrm{M}$ ) on the level of oxygenase activity of COX-1 in platelets. The Figure shows the average rate of change of Resorufin formation. The data represents the means of 12 measurements

is dependent on their activation level. The nonstimulated (resting) blood platelets lacked any free AA and therefore blocked the COX. After activation, an intracellular $\mathrm{Ca}^{2+}$ flux activated both enzymes: phospholipases $\mathrm{C}$ and $\mathrm{A}_{2}$, which hydrolyse membrane phospholipids and release free AA [33].

The exogenous arachidonic acid is able to cause irreversible platelet aggregation, as it can be rapidly incorporated into membrane phospholipids, primarily phosphatidylcholine and phosphatidylinositol, by arachidonoyl coenzyme A synthetase. Our current studies have demonstrated that flavonolignans are able to inhibit platelet aggregation induced by extracellular AA. This suggests the ability of the tested flavonolignans to block the arachidonic acid metabolism pathway in blood platelets. Additionally, in probes pre-incubated with flavonolignans we also observed inhibition of the formation of COX pathway metabolites: MDA and thromboxane $\mathrm{A}_{2}$. Following this, we determined the direct effect of flavonolignans on COX activity and saw that silychristin and silybin have very strong inhibitory properties with low $\mathrm{IC}_{50}$ values $(35 \mu \mathrm{M}$ and $60 \mu \mathrm{M}$ respectively).

The final step of our study, to explain the potential mechanism of obtained experimental results, was to characterise the interaction between selected flavonolignans and COX structure using bioinformatic ligand docking. In silico docking methods have undergone significant developments and improvements over the last few years [34]. Docking software computes the best conformation and placement of ligands in a receptor structure. For our study, we used the AutoDock Vina programme, which combines some of the advantages of knowledge-based potentials and empirical scoring functions. It extracts empirical information from both the conformational preferences of the receptor-ligand complex and from experimental affinity measurements [15].
The results obtained from the docking demonstrated the potential direct mechanism of interaction between flavonolignans (silychristin and silybin) and the active COX centre. This interaction block entry to the COX loop where is located Tyr385 residue. Tyr385 generates a tyrosyl radical in the cyclooxygenase active site which is abstracts the pro-S hydrogen atom from carbon-13 of AA, initiating the cyclooxygenase reaction [31, 35].

Acetylsalicylic (ASA) acid was the first cyclooxygenase inhibitor to be recognized and introduced to clinical use, and has been shown to be effective in reducing cardiovascular disease conditions associated with thrombosis, and increased blood platelet activation. The molecular mechanism of this inhibition is ASA's ability to cause irreversible acetylation of the platelet cyclooxygenase, with serine residue of: Ser529 of COX-1 and Ser516 of COX2. This acetylation results in blocking of the access of arachidonic acid to the active enzyme site, which leads to irreversible inhibition of the formation of both prothrombotic and pro-inflammatory mediators in blood platelets [36, 37]. ASA is the most popular substance in prevention of cardiovascular pathologies and has a wellestablished role in preventing thrombotic events in patients with recognized cardiovascular disease. Additionally, large-scale clinical studies conducted last years, demonstrated the effectiveness of ASA in the primary prevention of thrombotic events in men and women without diagnosed cardiovascular disease. In these studies, ASA significantly reduced the risk of a first myocardial infarction, stroke or death from cardiovascular causes $[38,39]$. However, ASA treatment is subject to risks from bleeding. Meta-analysis performed by Baigent et al. [8] demonstrated that long-term ASA administration increases the risk of major gastrointestinal and other extracranial bleedings by $0.5 \%$. In a study by De Berardis et al. [40], the risk of bleeding increased by 


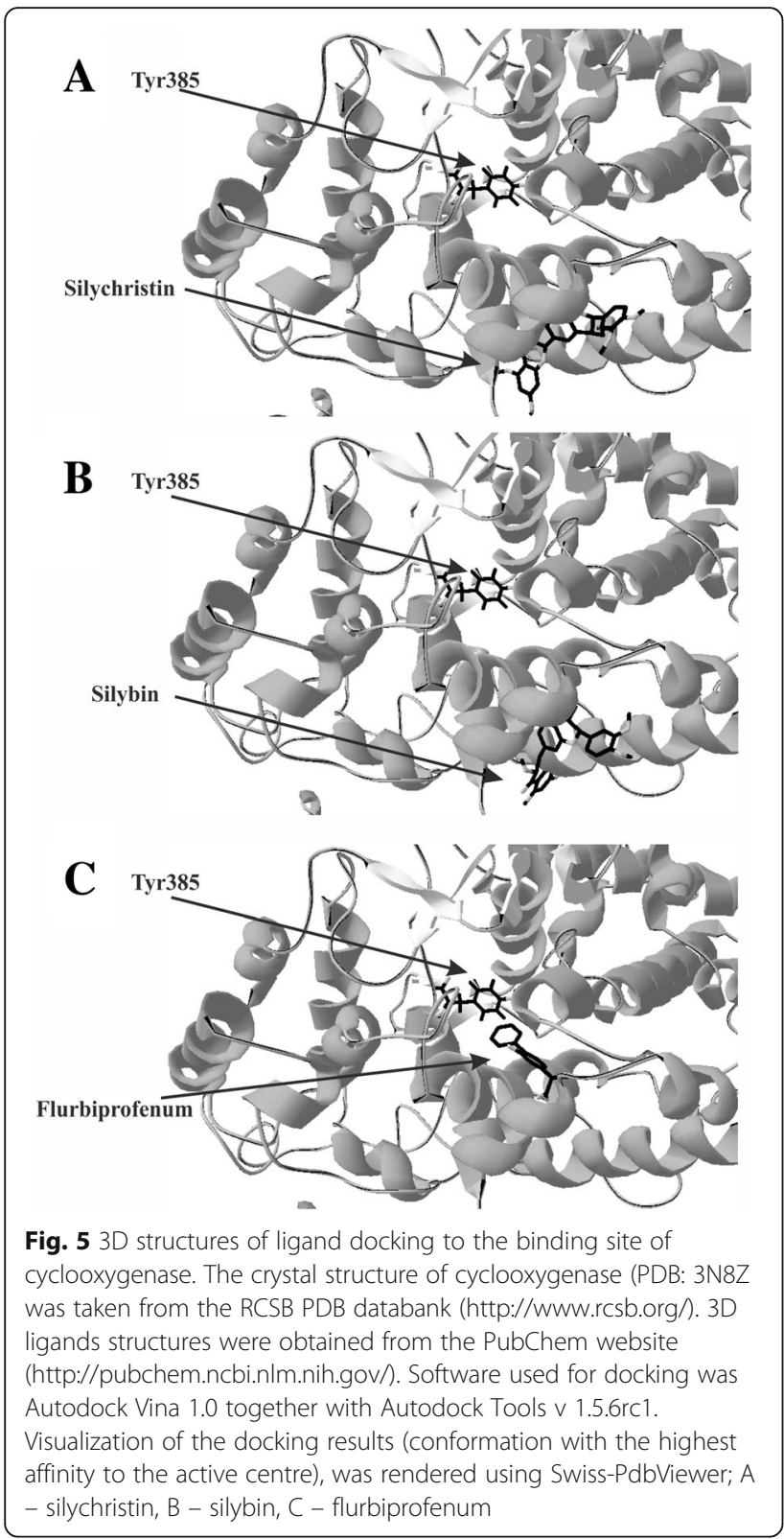

$2.5 \%$ in the ASA treatment group. ASA also increases the risk of haemorrhagic stroke [41]. These facts illustrate the necessity of ASA therapy to give proven clinical benefits, and the need for its assessment using precise estimation of benefits and risks, especially for rare events, such as intracranial haemorrhage [38]. For these reasons, all over the world incessant research is taking place on novel, therapeutically better, more effective anti-platelet agents that would have a significant effect on proper haemostatic stability, without adverse effects. Plants are a good source for isolating natural compounds without unfavourable side effects, capable of inhibiting the enzymes involved in cell signalling pathways and reducing blood platelets activation [42].

\section{Conclusions}

The results obtained from this study clearly present the potential role of flavonolignans, natural-origin compounds present in fruits of Milk thistle, an Asteraceae family plant (Silybum marianum, L. Gaernt.), as novel antiplatelet and anti-inflammatory agents. Since the 1970s, these compounds have been used as official medicine and are used in treatment of liver dysfunctions. Furthermore, as yet side effects from flavonolignans have not been found [43]. Additionally, in the last few years, novel forms of administration of Milk thistle extract have been discovered that have very high bioavailability (with plasma concentrations ranging from 60 to $70 \mu \mathrm{M}$ ) [44]. This corresponds with the concentrations of flavonolignans that have a biological effect in our study $(10-100 \mu \mathrm{M})$.

\section{Acknowledgements \\ None.}

\section{Funding}

This work was supported by grant B1611000001144.02 and grant 506/1136 from University of Lodz.

\section{Availability of data and materials}

All data are presented in the manuscript. Data sets used and/or analysed in this study are available from the corresponding author on reasonable request.

\section{Authors' contributions}

MB designed the study, did experimental work and analyses and drafted the manuscript. JSB collaborated in carrying out experimental work and analyses as well as performed a critical revision of the manuscript. Both authors read and approved the final manuscript.

\section{Ethics approval}

Analysis of the blood samples was performed under the guidelines of the Helsinki Declaration for Human Research, and approved by the Committee on the Ethics of Research in Human Experimentation at the University of Lodz (Resolution No. 16/KBBN-UŁ/II/2016). Blood samples were purchased from the Regional Center for Transfusion Medicine in Lodz (Poland). Each donor filled up and signed special questionnaire where he/she agree for commercial and scientific use of own blood by Regional Center for Transfusion Medicine.

Consent for publication Not applicable.

\section{Competing interests}

The authors declare that they have no competing interests.

\section{Publisher's Note}

Springer Nature remains neutral with regard to jurisdictional claims in published maps and institutional affiliations.

Received: 9 April 2017 Accepted: 31 July 2017

Published online: 10 August 2017

\section{References}

1. Sikora J, Kostka B. Blood platelets as pharmacological model. Post Biol Kom. 2005;232:561-70.

2. Palumbo $S$, Toscano CD, Parente L, Weigert R, Bosetti F. Time-dependent changes in the brain arachidonic acid cascade during cuprizone-induced demyelination and remyelination. Prostaglandins Leukot Essent Fat Acids. 2011;85:29-35. 
3. Ting HJ, Khasawneh FT. Platelet function and Isoprostane biology. Should isoprostanes be the newest member of the orphan-ligand family? J Biomed Sci. 2010;17:24.

4. Sobolewski C, Cerella C, Dicato M, Ghibelli L, Diederich M. The role of cyclooxygenase-2 in cell proliferation and cell death in human malignancies. Int J Cell Biol. 2010;2010:215158.

5. Polac I, Boryczka K, Bijak M, Borowiecka M, Wojsiat J, Stetkiewicz T. Acetylsalicylic acid and its application in disease prevention in postmenopausal women. Prz Menopauzalny. 2011;10:45-9.

6. Majek P, Reicheltova Z, Stikarova J, Suttnar J, Sobotkova A, Dyr JE. Proteome changes in platelets activated by arachidonic acid, collagen, and thrombin. Proteome Sci. 2010;8:56.

7. Warner TD, Nylander S, Whatling C. Anti-platelet therapy: cyclo-oxygenase inhibition and the use of aspirin with particular regard to dual anti-platelet therapy. Br J Clin Pharmacol. 2011;72:619-33.

8. Baigent C, Blackwell L, Collins R, Emberson J, Godwin J, Peto R, et al. Aspirin in the primary and secondary prevention of vascular disease: collaborative meta-analysis of individual participant data from randomised trials. Lancet. 2009;373:1849-60.

9. Dehmlow C, Murawski N, de Groot H. Scavenging of reactive oxygen species and inhibition of arachidonic acid metabolism by silibinin in human cells. Life Sci. 1996;58:1591-600.

10. Bijak M, Saluk J, Tsirigotis-Maniecka M, Komorowska H, Wachowicz B, Zaczynska $E$, et al. The influence of conjugates isolated from Matricaria chamomilla L. on platelets activity and cytotoxicity. Int J Biol Macromol. 2013;61:218-29.

11. Saluk-Juszczak J, Olas B, Nowak P, Staron A, Wachowicz B. Protective effects of D-glucaro-1,4-lactone against oxidative modifications in blood platelets. Nutr Metab Cardiovasc Dis. 2008;18:422-8.

12. Saluk-Juszczak J. A comparative study of antioxidative activity of calcium-Dglucarate, sodium-D-gluconate and D-glucono-1,4-lactone in a human blood platelet model. Platelets. 2010;21:632-40.

13. Walkowiak B, Kralisz U, Michalec L, Majewska E, Koziolkiewicz W, Ligocka A, et al. Comparison of platelet aggregability and P-selectin surface expression on platelets isolated by different methods. Thromb Res. 2000;99:495-502.

14. Olas B, Saluk-Juszczak J, Pawlaczyk I, Nowak P, Kolodziejczyk J, Gancarz R, et al. Antioxidant and antiaggregatory effects of an extract from Conyza canadensis on blood platelets in vitro. Platelets. 2006;17:354-60.

15. Trott O, Olson AJ. AutoDock Vina: improving the speed and accuracy of docking with a new scoring function, efficient optimization, and multithreading. J Comput Chem. 2010;31:455-61.

16. Saluk J, Bijak M, Ponczek MB, Nowak P, Wachowicz B. (1->3)-beta-d-Glucan reduces the damages caused by reactive oxygen species induced in human platelets by lipopolysaccharides. Carbohydr Polym. 2013;97:716-24.

17. Bijak M, Ponczek MB, Nowak P. Polyphenol compounds belonging to flavonoids inhibit activity of coagulation factor X. Int J Biol Macromol. 2014; 65:129-35.

18. Saluk J, Bijak M, Posmyk MM, Zbikowska HM. Red cabbage anthocyanins as inhibitors of lipopolysaccharide-induced oxidative stress in blood platelets. Int J Biol Macromol. 2015:80:702-9.

19. Sidhu RS, Lee JY, Yuan C, Smith WL. Comparison of cyclooxygenase-1 crystal structures: cross-talk between monomers comprising cyclooxygenase-1 homodimers. Biochemistry. 2010;49:7069-79.

20. Hanwell MD, Curtis DE, Lonie DC, Vandermeersch T, Zurek E, Hutchison GR. Avogadro: an advanced semantic chemical editor, visualization, and analysis platform. J Cheminform. 2012:4:17.

21. Sotriffer CA, Flader W, Winger RH, Rode BM, Liedl KR, Varga JM. Automated docking of ligands to antibodies: methods and applications. Methods. 2000; 20:280-91.

22. Rarey M, Kramer B, Lengauer T, Klebe G. A fast flexible docking method using an incremental construction algorithm. J Mol Biol. 1996;261:470-89.

23. Rodgers DW, Gamblin SJ, Harris BA, Ray S, Culp JS, Hellmig B, et al. The structure of unliganded reverse transcriptase from the human immunodeficiency virus type 1. Proc Natl Acad Sci U S A. 1995;92:1222-6.

24. Guex N, Peitsch MC. SWISS-MODEL and the Swiss-PdbViewer: an environment for comparative protein modeling. Electrophoresis. 1997;18:2714-23.

25. Bijak M, Kolodziejczyk-Czepas J, Ponczek MB, Saluk J, Nowak P. Protective effects of grape seed extract against oxidative and nitrative damage of plasma proteins. Int J Biol Macromol. 2012;51:183-7.
26. Bijak M, Nowak P, Borowiecka M, Ponczek MB, Zbikowska HM, Wachowicz B. Protective effects of (-)-epicatechin against nitrative modifications of fibrinogen. Thromb Res. 2012;130:123-8.

27. Saluk J, Bijak M, Kolodziejczyk-Czepas J, Posmyk MM, Janas KM, Wachowicz B. Anthocyanins from red cabbage extract - evidence of protective effects on blood platelets. Cent Eur J Biol. 2012;7:655-63.

28. Zbikowska HM, Antosik A, Szejk M, Bijak M, Olejnik AK, Saluk J, et al. Does quercetin protect human red blood cell membranes against gammairradiation? Redox Rep. 2014;19:65-71.

29. Zarghi A, Arfaei S. Selective COX-2 Inhibitors: A Review of Their StructureActivity Relationships. Iran J Pharm Res. 2011;10:655-83.

30. Aid S, Bosetti F. Targeting cyclooxygenases- 1 and -2 in neuroinflammation: Therapeutic implications. Biochimie. 2011;93:46-51.

31. Marnett LJ. Cyclooxygenase mechanisms. Curr Opin Chem Biol. 2000;4:545-52.

32. Huang JS, Ramamurthy SK, Lin X, Le Breton GC. Cell signalling through thromboxane A2 receptors. Cell Signal. 2004;16:521-33.

33. Rao GH, White JG. Role of arachidonic acid metabolism in human platelet activation and irreversible aggregation. Am J Hematol. 1985;19:339-47.

34. Mihasan M. What in silico molecular docking can do for the 'bench-working biologists'. J Biosci. 2012;37:1089-95.

35. Rouzer CA, Marnett LJ. Cyclooxygenases: structural and functional insights J Lipid Res. 2009:50:29-34.

36. Manious A, Tanner R, Shorr R, Limacher M. Use of Aspirin for Primary and Secondary Cardiovascular Disease Prevention in the United States, 20112012. J Am Heart Assoc. 2014;3:000989.

37. Patrono C. Aspirin: new cardiovascular uses for an old drug. Am J Med. 2001;110:62-5.

38. Nansseu JR, Noubiap JJ. Aspirin for primary prevention of cardiovascular disease. Thromb J. 2015;13:38.

39. Ittaman SV, VanWormer JJ, Rezkalla SH. The role of aspirin in the prevention of cardiovascular disease. Clin Med Res. 2014;12:147-54.

40. De Berardis G, Lucisano G, D'Ettorre A, Pellegrini F, Lepore V, Tognoni G, et al. Association of aspirin use with major bleeding in patients with and without diabetes. JAMA. 2012;307:2286-94.

41. He J, Whelton PK, Vu B, Klag MJ. Aspirin and risk of hemorrhagic stroke: a meta-analysis of randomized controlled trials. JAMA. 1998;280:1930-15.

42. Chua TK, Koh HL. Medicinal plants as potential sources of lead compounds with anti-platelet and anti-coagulant activities. Mini Rev Med Chem. 2006;6: 611-24.

43. Wesolowska O, Lania-Pietrzak B, Kuzdzal M, Stanczak K, Mosiadz D, Dobryszycki $P$, et al. Influence of silybin on biophysical properties of phospholipid bilayers. Acta Pharmacol Sin. 2007;28:296-306.

44. Parveen R, Baboota S, Ali J, Ahuja A, Vasudev SS, Ahmad S. Oil based nanocarrier for improved oral delivery of silymarin: In vitro and in vivo studies. Int J Pharm. 2011:413:245-53.

\section{Submit your next manuscript to BioMed Central and we will help you at every step:}

- We accept pre-submission inquiries

- Our selector tool helps you to find the most relevant journal

- We provide round the clock customer support

- Convenient online submission

- Thorough peer review

- Inclusion in PubMed and all major indexing services

- Maximum visibility for your research

Submit your manuscript at www.biomedcentral.com/submit 